\title{
Original Research \\ Identification of biomarkers and regulatory networks for cartilage damage patients
}

\author{
Baohong Liu ${ }^{1, *}$, Xingxing Xiao ${ }^{2}$ \\ ${ }^{1}$ State Key Laboratory of Veterinary Etiological Biology; Key Laboratory of Veterinary Parasitology of Gansu Province; Lanzhou Veterinary Research \\ Institute, Chinese Academy of Agricultural Sciences, 730046 Lanzhou, Gansu, China \\ ${ }^{2}$ Wenzhou Key Laboratory of Sanitary Microbiology; Key Laboratory of Laboratory Medicine, Ministry of Education; School of Laboratory Medicine \\ and Life Science, Wenzhou Medical University, 325035 Wenzhou, Zhejiang, China \\ *Correspondence: liubaohong@caas.cn (Baohong Liu) \\ Academic Editor: Josef Jampílek \\ Submitted: 15 December 2021 Revised: 13 January 2022 Accepted: 14 January 2022 Published: 11 February 2022
}

\begin{abstract}
Background: The aim of this study was to mine cartilage damage and regeneration-related biomarkers and identify the gene regulatory networks of cartilage damage. Methods: A gene expression data set (GSE129147) containing damaged and control samples collected from the knee of the same patients was employed. R package limma was used to identify differentially expressed genes (DEGs), and clusterProfiler was performed for the GO and KEGG functional enrichment analysis. Cytoscape plug-ins of CytoHubba and MCODE were applied to investigate protein-protein interaction (PPI) network, modules, and hub genes. Results: We identified 422 DEGs that were involved in skeletal system development, bone development, ossification, mesenchyme development, mesenchymal cell differentiation, connective tissue development, osteoblast differentiation, and extracellular matrix. We dug out 30 hub genes, identified three PPI modules, and constructed a miRNA regulatory network for DEGs. The miRNAs of the DEGs were predicted by miRNet, and the miRNA-mRNA network displayed some important miRNAs such as miR-335-5p, miR-92a-3p, and miR-98-5p. Conclusions: Collectively, these results have the potential to clarify the mechanism of cartilage damage and to assist us in discovering the damage and repair-related biomarkers.
\end{abstract}

Keywords: Cartilage damage; Protein-protein interaction (PPI) network; miRNA; Biomarkers

\section{Introduction}

In the joint, the surface of the connecting bone is covered with a layer of articular cartilage. The articular cartilage can absorb and buffer forces between the joints to the maximum extent. Cartilage damage, one of the major reasons for disability in the elderly [1], can occur in knees, hips, ankles, and elbows. Slight cartilage injuries may get better on their own in a few weeks, while severe damage may eventually require surgery. Because of the lack of selfregeneration for the damaged cartilage, it is essential to understand the molecular mechanisms in the progression of cartilage damage $[2,3]$. Some progress has been made in understanding the mechanisms of cartilage matrix degradation, and has promoted the progressive remodeling of the affected joints [4,5]. It is reported that the complex network of signaling molecules can fine-tune the cartilage differentiation $[6,7]$, so mining the molecular changes of damaged cartilage from a systematic level may shed light on the discovery of specific therapeutic targets.

Microarray technology has been extensively used in identifying disease-related biomarkers [8,9]. Besides mining the cartilage damage and regeneration-related mRNAs, emerging evidence indicates that miRNAs may also play an indispensable role in the network of regulating cartilage development $[10,11]$. MiRNAs are found to comprehen- sively modulate cartilage development by establishing an interaction network with target genes, transcription factors, and cytokines [12]. In this study, we performed a series of bioinformatics analyses to a public microarray data containing the cartilage-damaged samples and their corresponding control samples for the sake of revealing the mechanism of cartilage damage and mining cartilage damage and repair-related mRNA, miRNA biomarkers and their regulatory network.

\section{Materials and methods}

\subsection{Microarray process}

Microarray data GSE129147 was downloaded from the Gene Expression Omnibus database (www.ncbi.nlm.n ih.gov/geo). There are 19 samples containing 10 male patients with knee focal chondral defect and 9 undamaged regions of cartilage from the same patients. The array contains 49,395 probe sets in which 46,879 are mapped to at least one gene. We combined the probe sets mapping to one identical gene by maintaining the probe set that is most often associated with the highest expression level [13]. In total, 18,837 genes were achieved. The data set was quantile normalized by the function of normalizeQuantiles in $\mathrm{R}$ package limma (Fig. 1A) [14]. 
A

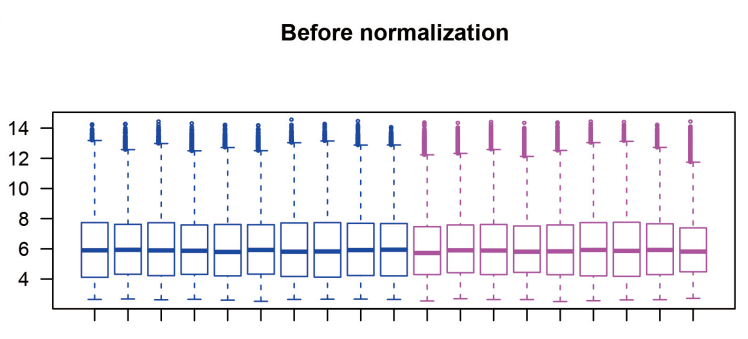

After normalization

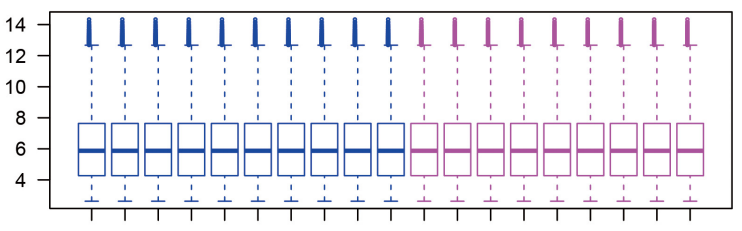

IIIIIIIDIDIm
B

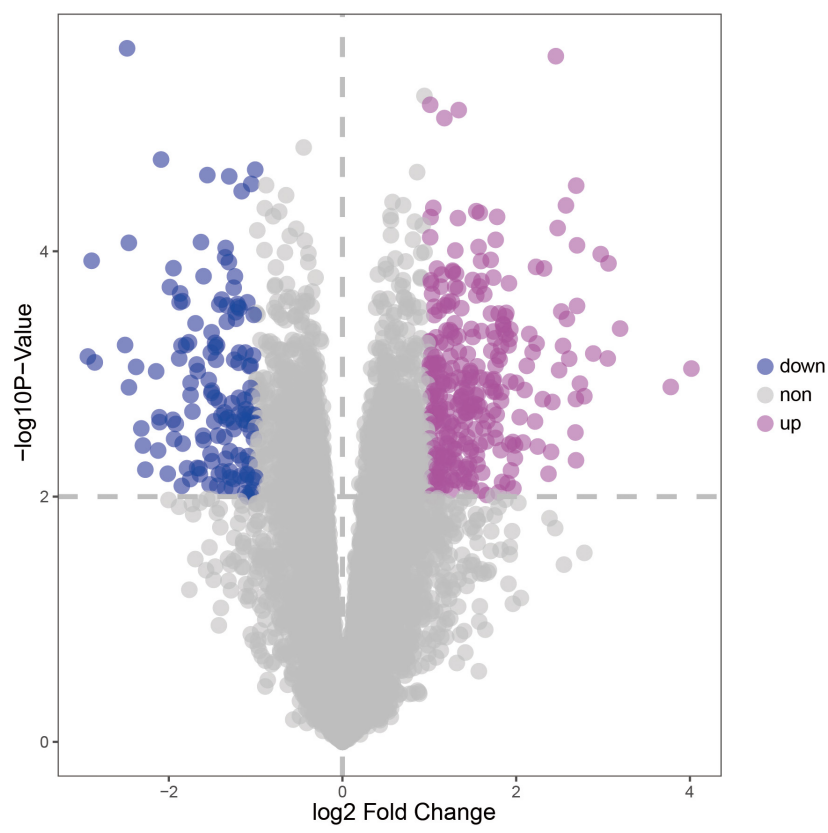

Fig. 1. The boxplot and volcano plot for genes. (A) The boxplot for samples before normalization and after normalization. (B) The volcano plot for DEGs.

\subsection{Differential expression analysis}

DEGs were identified by limma package in R [14]. A contrast matrix was constructed to distinguish samples from two groups. Then the linear modeling approach was implemented using function of $\mathrm{lmFit}$ and the empirical Bayes statistics was implemented by eBayes to identify DEGs. An adjusted $p$ value $<0.01$ and $\mid \log 2$ (fold-change) $\mid>1$ were used as the cut-off criteria.

\subsection{Gene functional enrichment analysis}

GO term and KEGG pathway functional enrichment analyses for DEGs were implemented by the R package clusterProfiler [15]. The Benjamini-Hochberg method adjusted $p$ value $<0.05$ was used as the cut-off criteria.

\subsection{Protein-protein interaction (PPI) network construction}

The PPI network was downloaded from the Search Tool for the Retrieval of Interacting Genes (STRING) database (https://string-db.org) [16]. The DEGs were inputted into the database under the default parameters, and the sub-PPI network for DEGs was obtained. Cytoscape [17] was used to visualize and analyze the DEGs-related PPI network. Hub genes of the network were identified by Cytoscape plug-in CytoHubba [18], and another plugin named MCODE was used to identify the PPI modules [19].

\subsection{Prediction of upstream miRNAs for DEGs}

Upstream miRNAs of DEGs were predicted using the miRNet database, which is an easy-to-use tool for miRNAassociated studies [20]. The DEGs were submitted to the database, and "Organism-H.sapiens" and "Tissue-Bone" were set as selection criteria.

\section{Results}

\subsection{DEG identification and functional enrichment analysis}

A total of 422 DEGs were identified, compared with the undamaged samples (Fig. 1B). Functional enrichment of GO terms showed that these DEGs were involved in the extracellular matrix $(\mathrm{ECM})\left(\right.$ adjusted $p$ value $=1.16 \times 10^{-11)}$, skeletal system development (adjusted $p$ value $=1.69 \times$ $10^{-10}$ ), bone development (adjusted $p$ value $=9.74 \times$ $10^{-8}$ ), ossification (adjusted $p$ value $=2.02 \times 10^{-7}$ ), mesenchyme development (adjusted $p$ value $=1.72 \times 10^{-5}$ ), mesenchymal cell differentiation (adjusted $p$ value $=2.14$ $\times 10^{-5}$ ), connective tissue development (adjusted $p$ value $=2.14 \times 10^{-5}$ ), and osteoblast differentiation (adjusted $p$ value $=2.14 \times 10^{-5}$ ). The KEGG pathway enrichment revealed the DEGs were engaged in the PI3K-Akt signaling pathway (adjusted $p$ value $=6.96 \times 10^{-3}$ ), ECM-receptor interaction (adjusted $p$ value $=1.13 \times 10^{-2}$ ), and rheumatoid arthritis (adjusted $p$ value $=3.85 \times 10^{-2}$ ) (Fig. 2).

\subsection{PPI network construction, hub genes, and protein-protein interaction modules identification}

The PPI network was built for the DEGs using the STRING database with 353 nodes and 1565 edges (see Fig. 3A). The degrees of the network were power-law distributed, which indicated that the network was scale free (see Fig. 3B and C). CytoHubba was applied to identify the hub genes in the network by gene degrees. We selected the top 30 genes with high degrees, which were MMP2, TGAM, 


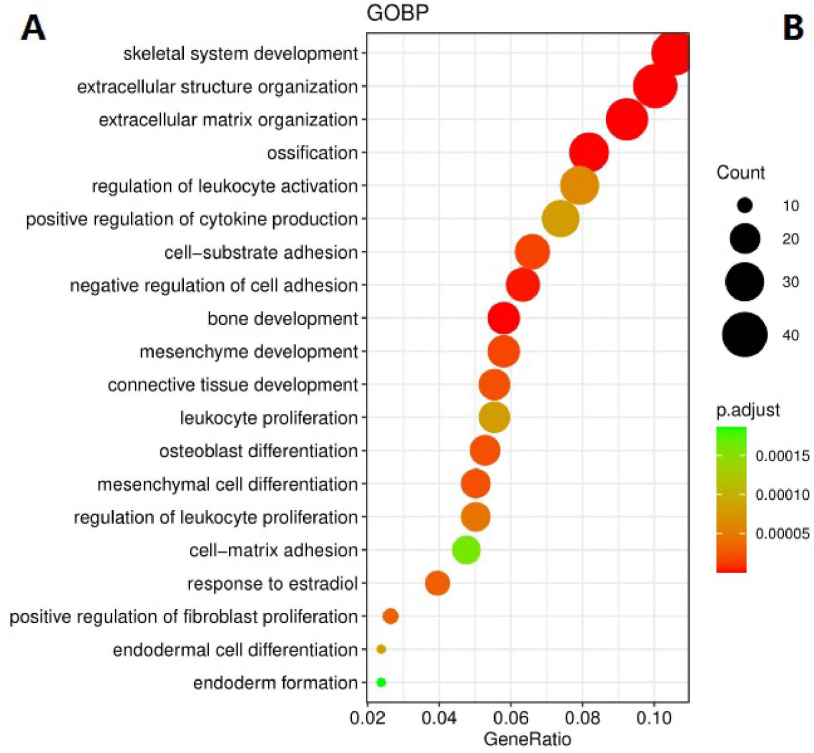

C

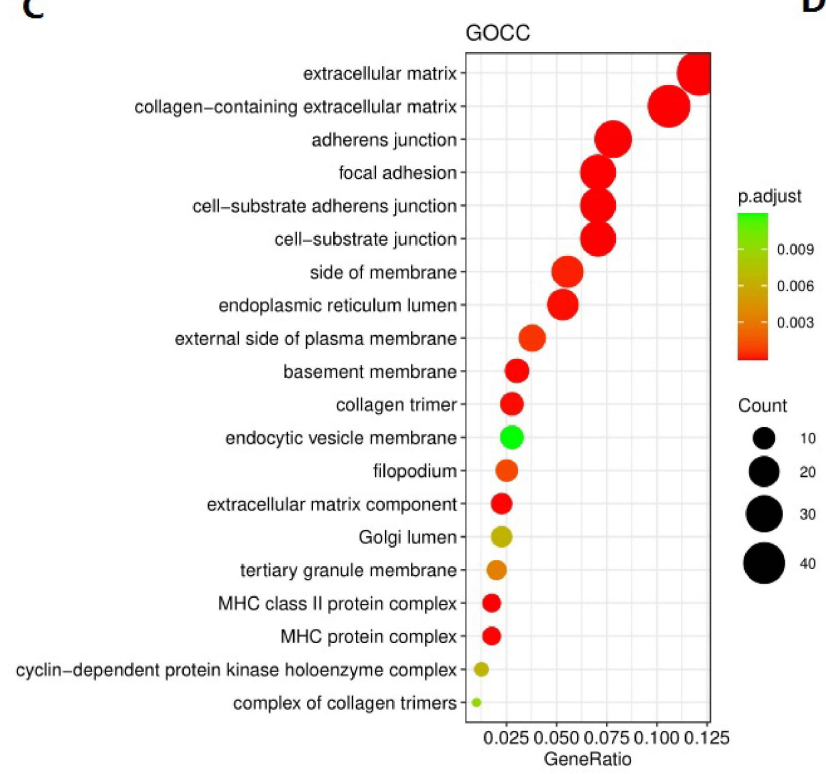

B

D
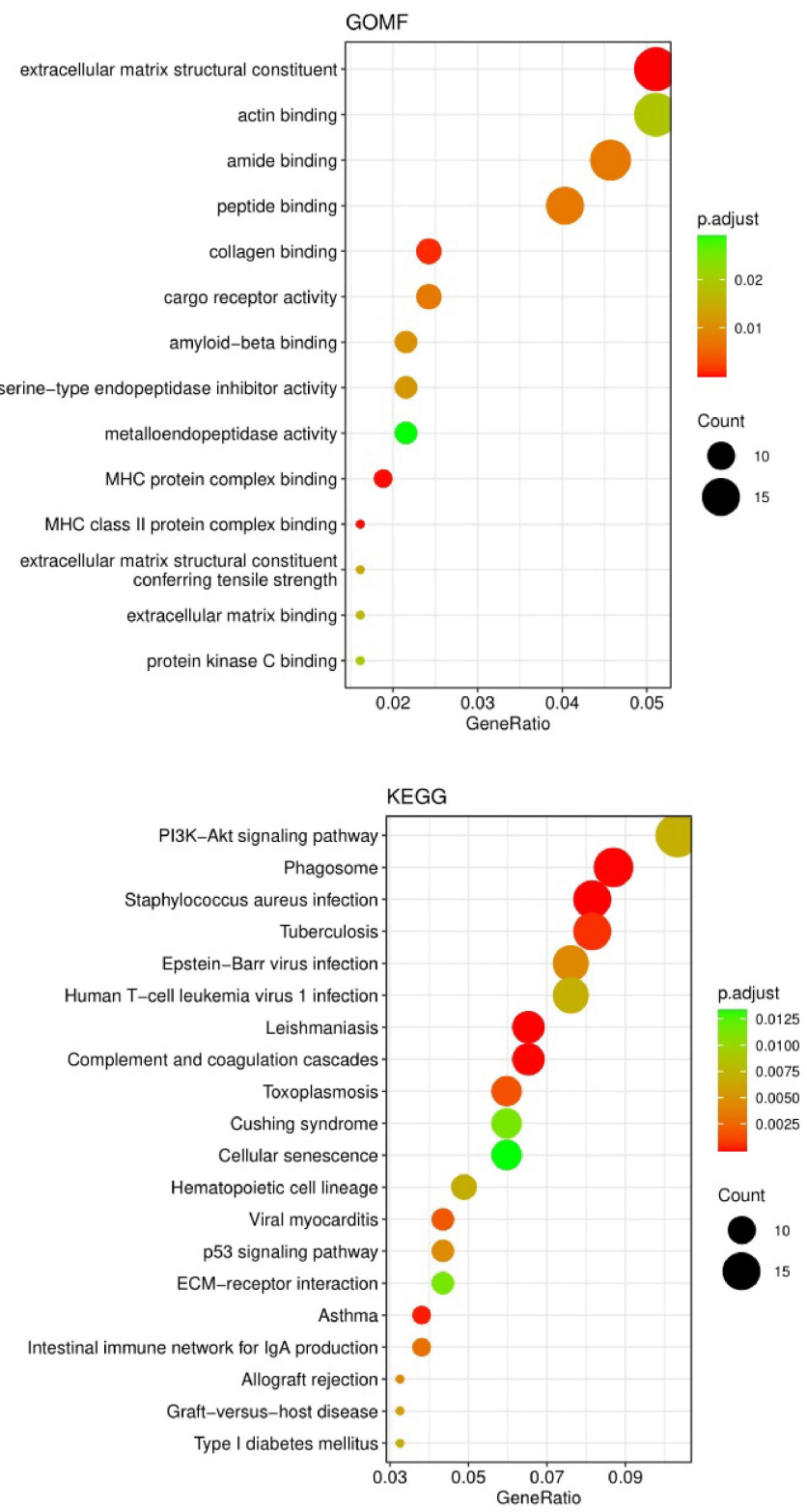

Fig. 2. The dot plot of the top 20 functional enrichment results. (A) Biological process. (B) Molecular function. (C) Cellular component. (D) KEGG pathway.

MYC, CCND1, COL1A1, CXCR4, ESR1, EZH2, CDK1, TYROBP, C3AR1, LOX, PTGS2, ITGB2, KIF11, CCNB1, $C 1 Q B, K I F 20 A, C Y B B, C D K N 3, T Y M S, P D G F R B, T O P 2 A$, POSTN, MMP14, COL5A1, FCGR3A, FCGR2B, WNT5A and $C D K N 2 A$ (see Table 1). The PPI modules were identified by Cytoscape plug-in MCODE. We selected three densely connected modules (see Fig. 4). Genes in module 1 were involved in the cell cycle (see Fig. 4A); genes module 2 were involved in the immune response related functions (see Fig. 4B); and genes in module 3 were related to extracellular matrix-related functions (see Fig. 4C), such as extracellular matrix organization, ECM-receptor interaction, focal adhesion, and collagen catabolic process.

\section{3 miRNAs prediction for the DEGs}

Using the miRNet database, we predicted 57 miRNAs, targeting the 442 DEGs. Within the 57 miRNAs, there were 48 miRNAs targeting the 30 hub DEGs. The regulatory network of DEGs and their upstream miRNAs was built using Cytoscape. Fig. 5A showed that mir-335-5p (degree: 82), mir-124-3p (degree: 68), mir-16-5p (degree: 38), mir-1925p (degree: 38), let-7b-5p (degree: 30), mir-92a-3p (degree: 30 ), mir-21-5p (degree: 23 ), mir-98-5p (degree: 22 ), and mir-17-5p (degree: 20 ) targeted no less than 20 genes respectively. Additionally, gene MYC (degree: 44) was regulated by most of the miRNAs in the whole miRNADEG regulatory network. Fig. 5B showed the regulatory network for hub DEGs and their upstream miRNAs. Addi- 

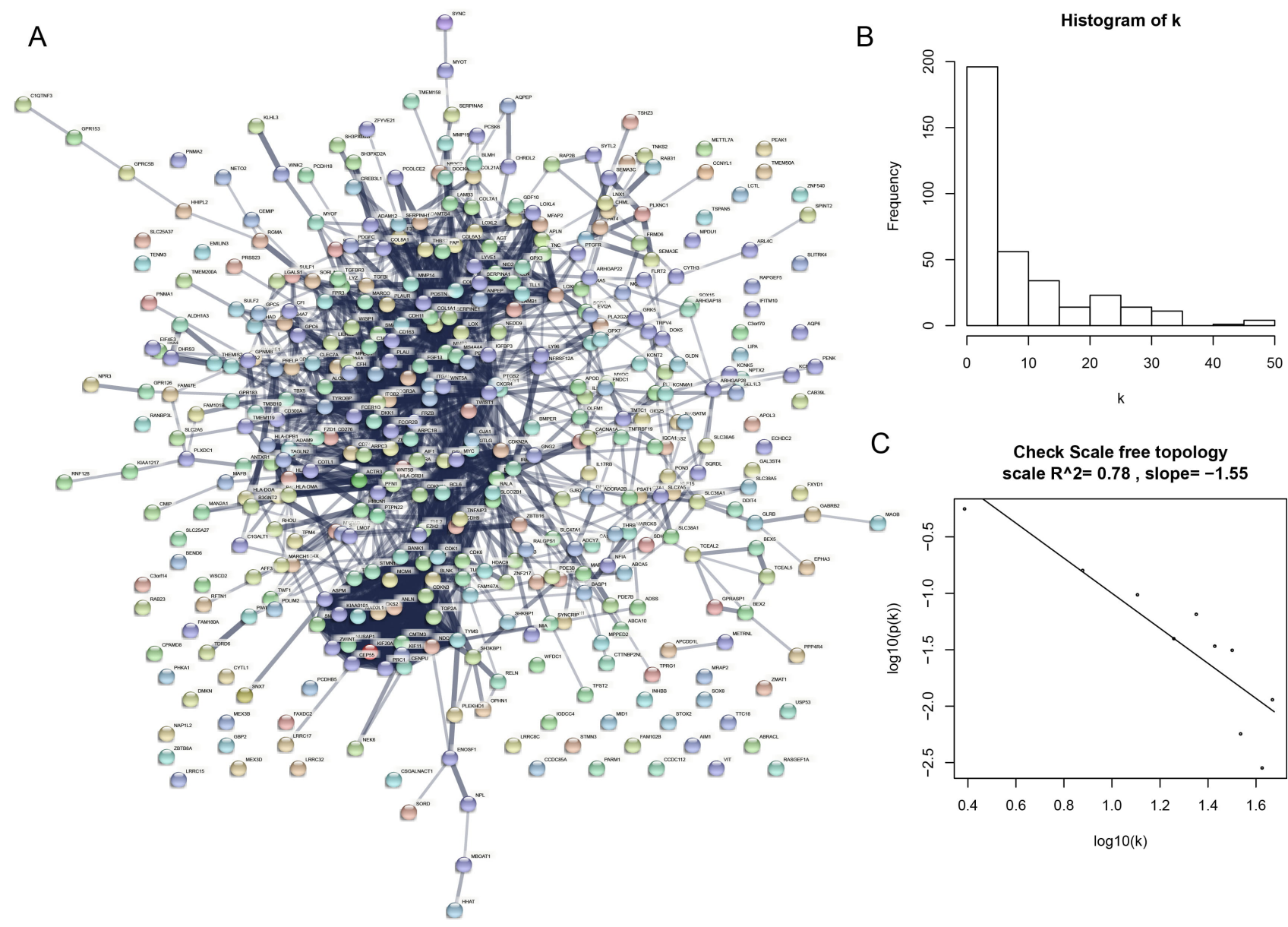

C

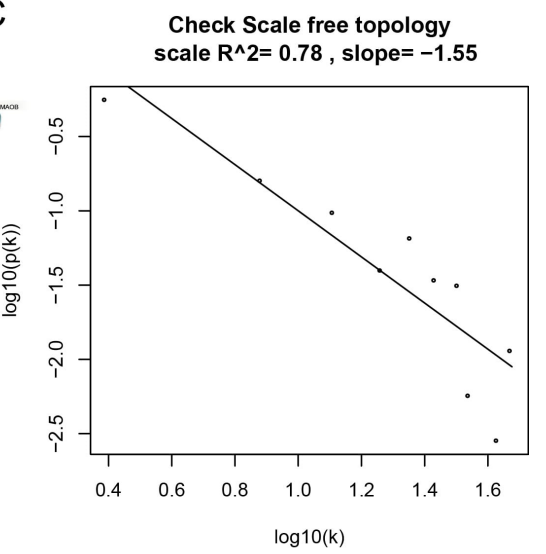

Fig. 3. The PPI network and its characteristics. (A) The PPI network from STRING database. (B) The node degree distribution for the PPI network. (C) The scale-free distribution of the node degree in the PPI network.

tionally, genes of $M Y C$ (degree: 44), CCNDI (degree: 17), and $E Z H 2$ (degree: 11) were targeted by at least 10 different miRNAs. MiRNAs of mir-335-5p (degree: 7), mir-34a-5p (degree: 7), and mir-92a-3p (degree: 7) were regulated by at least seven different genes (Fig. 5).

\section{Discussion}

We compared the gene expression profiles between damaged and non-damaged knee cartilage, and revealed the differences between them from a system biology level. We screened out 422 DEGs that were involved in extracellular matrix, ossification, osteoblast differentiation, and mesenchyme development, suggesting that these DEGs played important roles in the occurrence of cartilage damage. The PPI network was constructed using the STRING database, and the topological analysis showed that the node degree of the network was subject to the power-law distribution, which is a typical characteristic for a biological network [21]. Thirty DEGs with high degrees identified in the PPI network may play a pivotal role and modulate the functions of this network. Three modules were identified in the PPI network, and the 30 hub genes were highlighted in the mod- ules.

Genes in module1 were involved in cell cycles, and there were eight hub genes including $C C N B 1, C D K 1$, CDKN3, EZH2, KIF11, KIF20A, TOP2A, and TYMS. Among them, $C D K 1$ (cyclin-dependent kinase 1) plays a key role in controlling the cell-cycle process for the eukaryotes. It can interact with multiple interphase cyclins to promote G2-M transition, and it regulates the G1 progress and G1-S transition [22,23]. KIF11 (kinesin family member 11) is an essential molecular motor protein for mitosis. It can support cell proliferation by mediating centrosome separation and formation of the bipolar mitotic spindle [24-26].

Genes in module2 were associated with immune response related categories. The immune system is involved in the process of tissue injury, which indicates that tissue, organ, or appendage regeneration might be influenced by cartilage damage [27]. There were five hub genes including ClQB, C3AR1, CXCR4, FCGR3A and TYROBP in this module. Among the five hub genes, CXCR4 is an important molecular in cartilage degradation and the CXCL12/CXCR4 axis plays a pivotal role in injury and cartilage repair by acting as a chemo attractant of cells involved in inflammation and stem cell migration [28-30]. 
Table 1. The top 30 hub genes in the PPI network.

\begin{tabular}{|c|c|c|c|c|}
\hline Symbol & Description & Degree & $\log 2(\mathrm{FC})$ & $p$ value \\
\hline MMP2 & Matrix metallopeptidase 2 & 49 & 1.96 & $3.59 \times 10^{-3}$ \\
\hline ITGAM & Integrin, alpha $\mathrm{M}$ & 48 & 1.76 & $8.08 \times 10^{-5}$ \\
\hline MYC & BHLH Transcription Factor & 47 & 1.23 & $3.23 \times 10^{-3}$ \\
\hline CCND1 & Cyclin D1 & 46 & 1.42 & $1.45 \times 10^{-3}$ \\
\hline COL1A1 & Collagen Type I Alpha 1 Chain & 43 & 3.78 & $1.28 \times 10^{-3}$ \\
\hline CXCR4 & C-X-C Motif Chemokine Receptor 4 & 35 & 1.10 & $8.80 \times 10^{-3}$ \\
\hline ESR1 & Estrogen Receptor 1 & 35 & -1.11 & $2.57 \times 10^{-3}$ \\
\hline $\mathrm{EZH} 2$ & Enhancer Of Zeste 2 Polycomb Repressive Complex 2 Subunit & 34 & 1.10 & $1.34 \times 10^{-3}$ \\
\hline CDK1 & Cyclin Dependent Kinase 1 & 34 & 1.46 & $1.13 \times 10^{-3}$ \\
\hline TYROBP & Transmembrane Immune Signaling Adaptor TYROBP & 33 & 1.30 & $5.07 \times 10^{-3}$ \\
\hline C3AR1 & Complement C3a Receptor 1 & 33 & 1.92 & $1.82 \times 10^{-4}$ \\
\hline LOX & Lysyl Oxidase & 33 & 1.15 & $1.09 \times 10^{-3}$ \\
\hline PTGS2 & Prostaglandin-Endoperoxide Synthase 2 & 32 & 1.90 & $7.77 \times 10^{-3}$ \\
\hline ITGB2 & Integrin Subunit Beta 2 & 32 & 1.67 & $2.43 \times 10^{-3}$ \\
\hline KIF11 & Kinesin Family Member 11 & 32 & 1.12 & $1.82 \times 10^{-3}$ \\
\hline CCNB1 & Cyclin B1 & 32 & 1.03 & $2.08 \times 10^{-3}$ \\
\hline C1QB & Complement C1q B Chain & 30 & 1.74 & $4.51 \times 10^{-4}$ \\
\hline KIF20A & Kinesin Family Member 20A & 30 & 1.21 & $3.07 \times 10^{-3}$ \\
\hline CYBB & Cytochrome B-245 Beta Chain & 29 & 2.46 & $2.57 \times 10^{-6}$ \\
\hline CDKN3 & Cyclin Dependent Kinase Inhibitor 3 & 29 & 1.57 & $1.94 \times 10^{-4}$ \\
\hline TYMS & Thymidylate Synthetase & 29 & 1.80 & $3.22 \times 10^{-4}$ \\
\hline PDGFRB & Platelet Derived Growth Factor Receptor Beta & 28 & 1.16 & $2.40 \times 10^{-3}$ \\
\hline TOP2A & DNA Topoisomerase II Alpha & 28 & 1.66 & $3.56 \times 10^{-3}$ \\
\hline POSTN & Periostin & 27 & 4.02 & $9.05 \times 10^{-4}$ \\
\hline MMP14 & Matrix Metallopeptidase 14 & 27 & 1.74 & $1.64 \times 10^{-4}$ \\
\hline COL5A1 & Collagen Type V Alpha 1 Chain & 27 & 1.09 & $2.16 \times 10^{-3}$ \\
\hline FCGR3A & Fc Fragment Of IgG Receptor IIIa & 26 & 2.57 & $4.23 \times 10^{-5}$ \\
\hline FCGR2B & Fc Fragment Of IgG Receptor IIb & 26 & 1.48 & $4.01 \times 10^{-3}$ \\
\hline WNT5A & Wnt Family Member 5A & 26 & 1.60 & $4.47 \times 10^{-4}$ \\
\hline CDKN2A & Cyclin Dependent Kinase Inhibitor 2A & 26 & 1.05 & $4.43 \times 10^{-5}$ \\
\hline
\end{tabular}

Module 3 enriched genes involved in ECM-related functions. Four hub genes including COL1A1, COL5A1, POSTN and PTGS2 were highlighted in this module. COL1A1 and COL5A1 are collagen genes. The expression of COL1A1 encoding a pro-alphal chain of type I collagen is abundant in bone. The mutations on COL1A1 are the major cause of osteogenesis imperfecta [31]. COL5A1 is the minor component of connective tissue and encodes the alpha chain of type $\mathrm{V}$ collagen. An animal study showed that the dysfunction of COL5A1 can generate an abnormal joint phenotype, such as joint laxity and early-onset osteoarthritis [32]. The extracellular matrix loss can lead to destruction of cartilage through uncontrolled production of matrixdegrading enzymes [33]. POSTN encodes a secreted extracellular matrix protein, playing a part in tissue development and regeneration. It can solidify connective tissues by crosslinking to other ECM proteins [31]. POSTN-null mice showed defective collagen crosslinks and decreases resistance to mechanical stress [34]. POSTN is re-expressed in fibrous tissues formed after injury and recruits mesenchymal cells by interacting with integrin, which is followed by tissue repair [35]. Therefore, POSTN has a crucial role in tissue repair. POSTN mRNA level was significantly higher in the OA cartilage than that in the controls [36]. In our analysis, $P O S T N$ is an up-regulated DEG $(\log \mathrm{FC}=4.02 ; p$ $=9.05 \times 10^{-4}$ ), indicating that the POSTN may start the function of tissue repair after the cartilage damage. PTGS2 is an important gene for osteoarthritis. Dranitsina et al. [37] reported that the expression of TGFB1 and PTGS2 genes changed in rats' knee joint cartilage under osteoarthrosis, suggesting the development of pathological processes in cartilage tissue, especially in degeneration and inflammation tissues.

Furthermore, upstream regulatory miRNAs were predicted for the DEGs. Mir-335-5p can regulate bone development to promote osteogenic differentiation [38]. In our study, the target mRNAs for miR-335 were mainly upregulated, which suggests that the expression level of miR- 

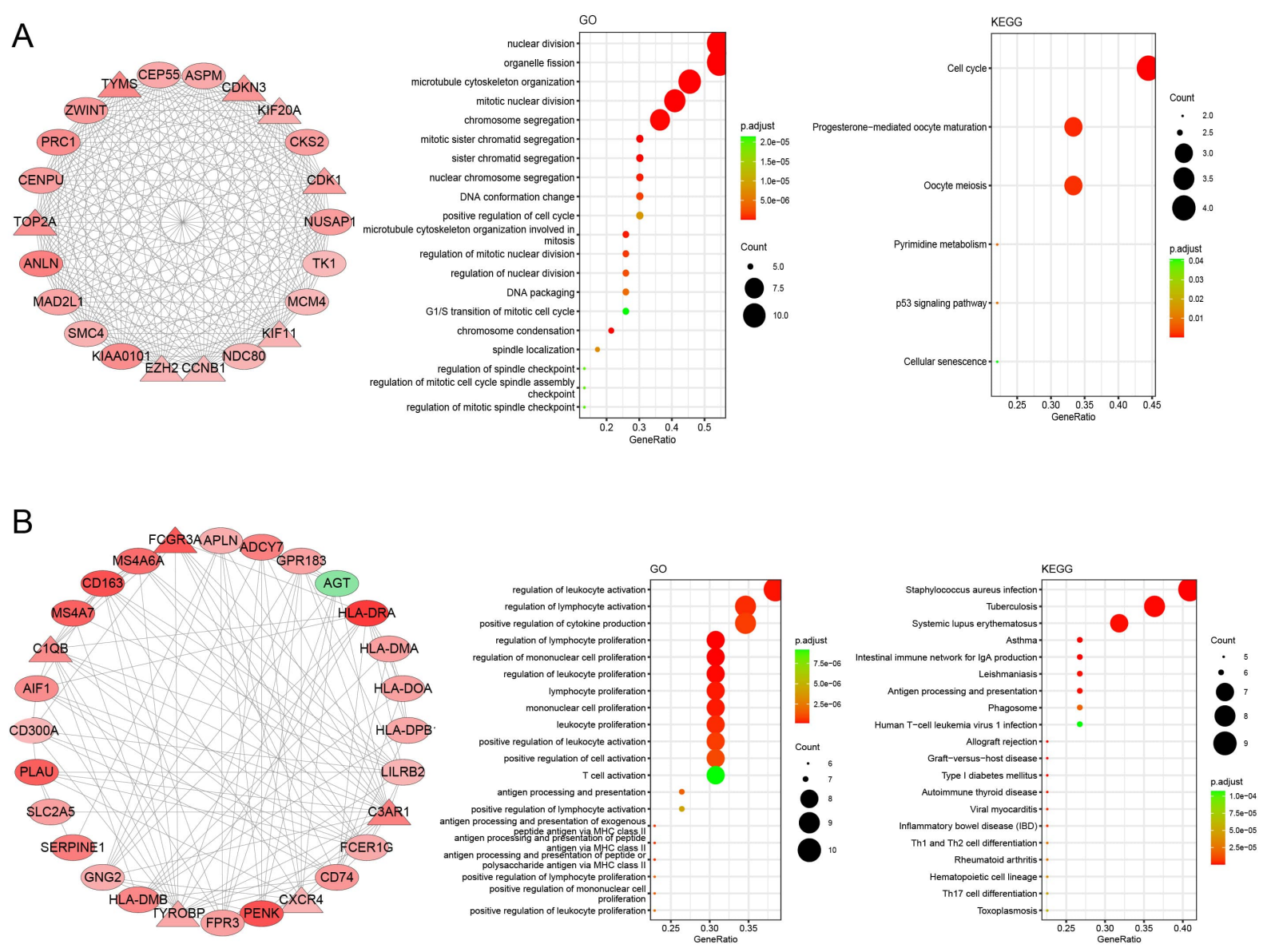

C
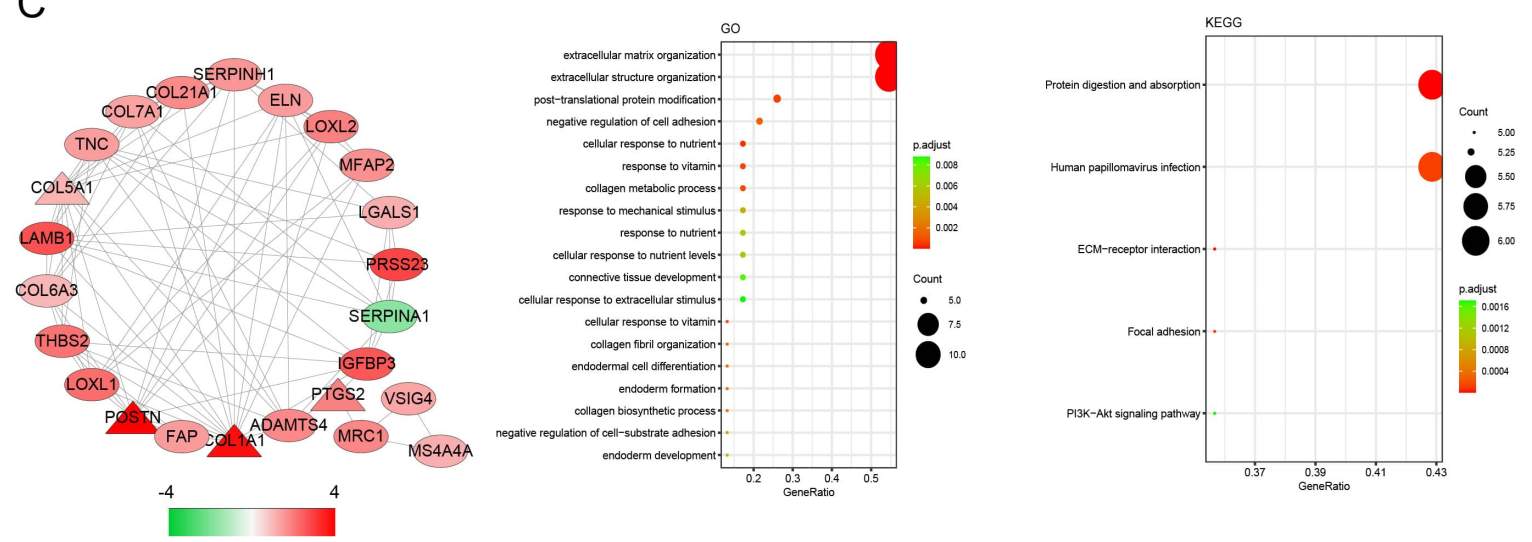

Fig. 4. Modules identified by MCODE and module functions by clusterProfiler. (A) Module 1 is involved in the cell cycle and included eight hub genes. (B) Module 2 is involved in the immune response and included five hub genes. (C) Module 3 is involved in extracellular matrix-related functions and included four hub genes. The colors represent the $\log 2$ fold change of genes. The nodes of triangle represent the hub genes identified by CytoHubba in PPI network.

335 may be down-regulated. It was reported that tissue damage or proinflammatory signals may cause downregulation of miR-335 and then activate the proliferative, migratory, and differentiation capacities of MSCs [39]. MiR-92a$3 p$ plays a key role in chondrogenesis and cartilage degradation, and Mao et al. [40] revealed that miR-92a-3p enhances cartilage development and prevents degradation by targeting $W N T 5 A$. In this study, WNT5A was significantly up-regulated with $\log 2$ fold change 1.60 and $p$ value $4.47 \times$ $10^{-4}$, which was in accordance with the results that when the cartilage was damaged, miR-92a-3p repressed the chondrogenic differentiation and reduced cartilage matrix synthesis by enhancing the expression of WNT5A [41]. MiR$98-5 \mathrm{p}$ is involved in the regulation of osteoblast differenti- 
A

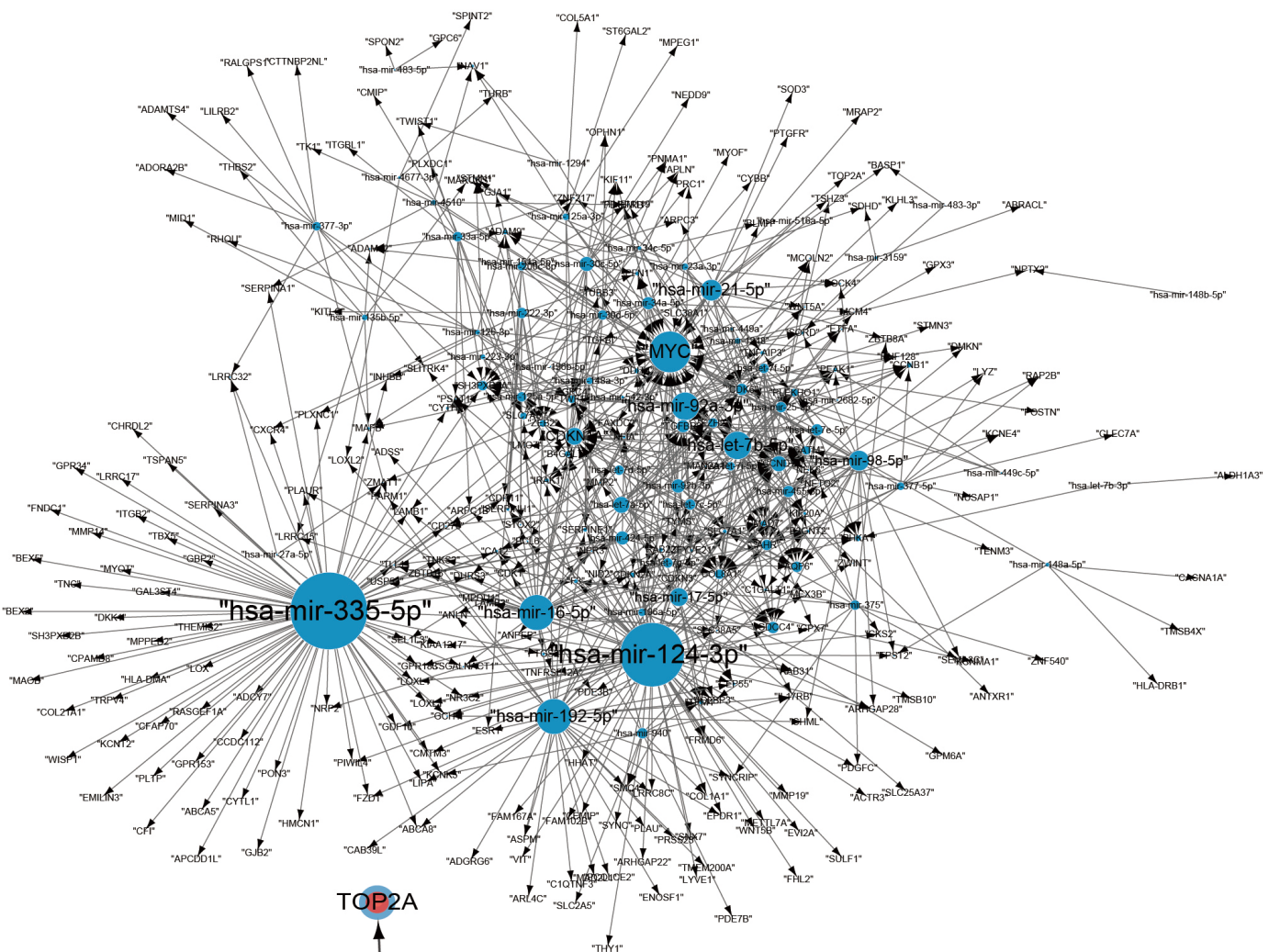

B

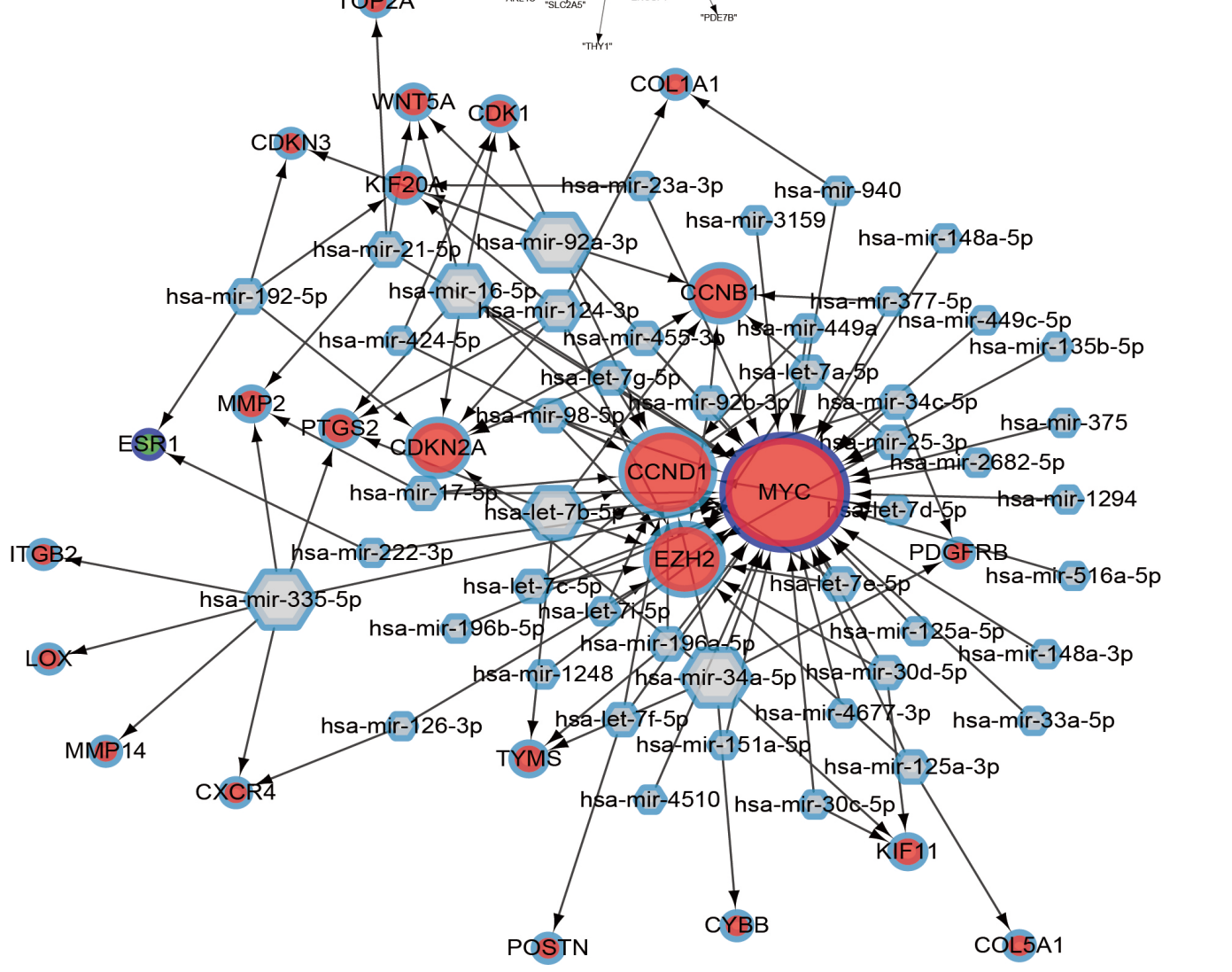

Fig. 5. The regulatory network of cartilage damage-related genes and miRNAs. (A) The DEGs-relate miRNA-target regulatory network (whole miRNA-DEG network). The size of the nodes represents their degree in the network. (B) The top 30 DEGs-related miRNA-target regulatory network (miRNA-hub DEG network). The circles represent the genes and the hexagons represent the miRNAs. Up-regulated genes are filled in red and down-regulated genes are filled in green. The size of the nodes represents their degree in the network. 
ation and promotes its differentiation through targeting and regulating $C K I P-1$ expression [42].

Although several important clusters and genes were identified in the cartilage damage patients, there were still some limitations for the current work. It is not available for additional microarray/RNA-seq data to validate the results in GSE129147. The mechanisms underlying the findings have not been thoroughly clarified and wet experiments such as qPCR and western blot in the mouse model or in clinical patients need to be carried out.

\section{Conclusions}

In this work, we analyzed a microarray data for cartilage-damaged patients, compared it with the controls, and identified three important modules, key genes and miRNAs related to the cartilage damage. Most of them were validated previously and related to the cartilage development or similar functions. All of these results need to be validated by more experiments.

\section{Author contributions}

BL conceived and designed the study, collected and analyzed the data, wrote and revised the manuscript. $\mathrm{XX}$ revised the manuscript.

\section{Ethics approval and consent to participate}

Not applicable.

\section{Acknowledgment}

The author thanks the researchers from Ankara Yildirim Beyazit University submitting the dataset to GEO.

\section{Funding}

This work was supported by the Innovative Special Project of Agricultural Sci-Tech (Grant No. CAASASTIP2014-LVRI-09).

\section{Conflict of interest}

The authors declare no conflict of interest.

\section{Data availability}

The GSE129147 was downloaded from GEO website of https:/www.ncbi.nlm.nih.gov/gds/?term=GSE129147.

\section{References}

[1] Curl WW, Krome J, Gordon ES, Rushing J, Smith BP, Poehling GG. Cartilage injuries: a review of 31,516 knee arthroscopies. Arthroscopy. 1997; 13: 456-460.

[2] Shah RN, Shah NA, Del Rosario Lim MM, Hsieh C, Nuber G, Stupp SI. Supramolecular design of self-assembling nanofibers for cartilage regeneration. Proceedings of the National Academy of Sciences of the United States of America. 2010; 107: 32933298.

[3] Wang M, Yuan Z, Ma N, Hao C, Guo W, Zou G, et al. Advances and Prospects in Stem Cells for Cartilage Regeneration. Stem Cells International. 2017; 2017: 4130607.
[4] Pap T, Korb-Pap A. Cartilage damage in osteoarthritis and rheumatoid arthritis-two unequal siblings. Nature Reviews. Rheumatology. 2015; 11: 606-615.

[5] Gupta PK, Das AK, Chullikana A, Majumdar AS. Mesenchymal stem cells for cartilage repair in osteoarthritis. Stem Cell Research \& Therapy. 2012; 3: 25.

[6] Cao L, Yang F, Liu G, Yu D, Li H, Fan Q, et al. The promotion of cartilage defect repair using adenovirus mediated Sox 9 gene transfer of rabbit bone marrow mesenchymal stem cells. Biomaterials. 2011; 32: 3910-3920.

[7] Mariani E, Pulsatelli L, Facchini A. Signaling pathways in cartilage repair. International Journal of Molecular Sciences. 2014; 15: 8667-8698.

[8] Kamel HFM, Al-Amodi HSAB. Exploitation of Gene Expression and Cancer Biomarkers in Paving the Path to Era of Personalized Medicine. Genomics, Proteomics \& Bioinformatics. 2017; 15: 220-235.

[9] Li H, Lu H. Transcriptome analyses identify key genes and potential mechanisms in a rat model of osteoarthritis. Journal of Orthopaedic Surgery and Research. 2018; 13: 319.

[10] Asahara H. Current Status and Strategy of microRNA Research for Cartilage Development and Osteoarthritis Pathogenesis. Journal of Bone Metabolism. 2016; 23: 121-127.

[11] Baek D, Lee K, Park KW, Suh JW, Choi SM, Park KH, et al. Inhibition of miR-449a Promotes Cartilage Regeneration and Prevents Progression of Osteoarthritis in in Vivo Rat Models. Molecular Therapy - Nucleic Acids. 2018; 13: 322-333.

[12] Shang J, Liu H, Zhou Y. Roles of microRNAs in prenatal chondrogenesis, postnatal chondrogenesis and cartilage-related diseases. Journal of Cellular and Molecular Medicine. 2013; 17: $1515-1524$.

[13] Liu BH, Cai JP. Identification of Transcriptional Modules and Key Genes in Chickens Infected with Salmonella enterica Serovar Pullorum Using Integrated Coexpression Analyses. BioMed Research International. 2017; 2017: 1-12.

[14] Smyth GK. Linear Models and Empirical Bayes Methods for Assessing Differential Expression in Microarray Experiments. Statistical Applications in Genetics and Molecular Biology. 2004; 3: $1-25$.

[15] Yu G, Wang L, Han Y, He Q. ClusterProfiler: an R package for comparing biological themes among gene clusters. Omics. 2012; 16: 284-287.

[16] Szklarczyk D, Franceschini A, Kuhn M, Simonovic M, Roth A, Minguez P, et al. The STRING database in 2011: functional interaction networks of proteins, globally integrated and scored. Nucleic Acids Research. 2011; 39: D561-D568.

[17] Shannon P, Markiel A, Ozier O, Baliga NS, Wang JT, Ramage D, et al. Cytoscape: a software environment for integrated models of biomolecular interaction networks. Genome Research. 2003; 13: 2498-2504.

[18] Chin C, Chen S, Wu H, Ho C, Ko M, Lin C. CytoHubba: identifying hub objects and sub-networks from complex interactome. BMC Systems Biology. 2014; 8: S11.

[19] Bader GD, Hogue CWV. An automated method for finding molecular complexes in large protein interaction networks. BMC Bioinformatics. 2003; 4: 2.

[20] Fan Y, Xia J. MiRNet-Functional Analysis and Visual Exploration of miRNA-Target Interactions in a Network Context. Methods in Molecular Biology. 2018; 1819: 215-233.

[21] Liu B. Differential Coexpression Network Analysis for Gene Expression Data. Methods in Molecular Biology. 2018; 1754: 155-165.

[22] Brown NR, Korolchuk S, Martin MP, Stanley WA, Moukhametzianov R, Noble MEM, et al. CDK1 structures reveal conserved and unique features of the essential cell cycle CDK. Nature Communications. 2015; 6: 6769. 
[23] Santamaría D, Barrière C, Cerqueira A, Hunt S, Tardy C, Newton $\mathrm{K}$, et al. Cdk1 is sufficient to drive the mammalian cell cycle. Nature. 2007; 448: 811-815.

[24] Kahn OI, Sharma V, González-Billault C, Baas PW. Effects of kinesin-5 inhibition on dendritic architecture and microtubule organization. Molecular Biology of the Cell. 2015; 26: 66-77.

[25] Mayer TU, Kapoor TM, Haggarty SJ, King RW, Schreiber SL, Mitchison TJ. Small molecule inhibitor of mitotic spindle bipolarity identified in a phenotype-based screen. Science. 1999; 286: 971-974.

[26] Sarli V, Giannis A. Targeting the kinesin spindle protein: basic principles and clinical implications. Clinical Cancer Research. 2008; 14: 7583-7587.

[27] Godwin JW, Brockes JP. Regeneration, tissue injury and the immune response. Journal of Anatomy. 2006; 209: 423-432.

[28] Chen P, Tao J, Zhu S, Cai Y, Mao Q, Yu D, et al. Radially oriented collagen scaffold with SDF-1 promotes osteochondral repair by facilitating cell homing. Biomaterials. 2015; 39: 114-123.

[29] Lu W, Shi J, Zhang J, Lv Z, Guo F, Huang H, et al. CXCL12/CXCR4 Axis Regulates Aggrecanase Activation and Cartilage Degradation in a Post-Traumatic Osteoarthritis Rat Model. International Journal of Molecular Sciences. 2016; 17: 1522.

[30] Thomas NP, Li P, Fleming BC, Chen Q, Wei X, Xiao-Hua P, et al. Attenuation of cartilage pathogenesis in post-traumatic osteoarthritis (PTOA) in mice by blocking the stromal derived factor 1 receptor (CXCR4) with the specific inhibitor, AMD3100. Journal of Orthopaedic Research. 2015; 33: 1071-1078.

[31] Horiuchi K, Amizuka N, Takeshita S, Takamatsu H, Katsuura $\mathrm{M}$, Ozawa $\mathrm{H}$, et al. Identification and characterization of a novel protein, periostin, with restricted expression to periosteum and periodontal ligament and increased expression by transforming growth factor beta. Journal of Bone and Mineral Research. 1999; 14: 1239-1249.

[32] Aro E, Salo AM, Khatri R, Finnilä M, Miinalainen I, Sormunen $\mathrm{R}$, et al. Severe Extracellular Matrix Abnormalities and Chondrodysplasia in Mice Lacking Collagen Prolyl 4-Hydroxylase Isoenzyme II in Combination with a Reduced Amount of Isoenzyme I. The Journal of Biological Chemistry. 2015; 290: 16964 16978.

[33] Pattoli MA, MacMaster JF, Gregor KR, Burke JR. Collagen and
Aggrecan Degradation is Blocked in Interleukin-1-Treated Cartilage Explants by an Inhibitor of iкB Kinase through Suppression of Metalloproteinase Expression. Journal of Pharmacology and Experimental Therapeutics. 2005; 315: 382-388.

[34] Norris RA, Damon B, Mironov V, Kasyanov V, Ramamurthi A, Moreno-Rodriguez $\mathrm{R}$, et al. Periostin regulates collagen fibrillogenesis and the biomechanical properties of connective tissues. Journal of Cellular Biochemistry. 2007; 101: 695-711.

[35] Kudo A. Periostin in fibrillogenesis for tissue regeneration: periostin actions inside and outside the cell. Cellular and Molecular Life Sciences. 2011; 68: 3201-3207.

[36] Chijimatsu R, Kunugiza Y, Taniyama Y, Nakamura N, Tomita T, Yoshikawa H. Expression and pathological effects of periostin in human osteoarthritis cartilage. BMC Musculoskeletal Disorders. 2015; 16: 215 .

[37] Korotkyi OH, Vovk AA, Dranitsina AS, Falalyeyeva TM, Dvorshchenko KO, Fagoonee $\mathrm{S}$, et al. The influence of probiotic diet and chondroitin sulfate administration on Ptgs2, Tgfb1 and Col2a1 expression in rat knee cartilage during monoiodoacetateinduced osteoarthritis. Minerva Medica. 2019; 110: 419-424.

[38] Zhang J, Tu Q, Bonewald LF, He X, Stein G, Lian J, et al. Effects of miR-335-5p in modulating osteogenic differentiation by specifically downregulating Wnt antagonist DKK1. Journal of Bone and Mineral Research. 2011; 26: 1953-1963.

[39] Tomé M, López-Romero P, Albo C, Sepúlveda JC, FernándezGutiérrez B, Dopazo A, et al. MiR-335 orchestrates cell proliferation, migration and differentiation in human mesenchymal stem cells. Cell Death and Differentiation. 2011; 18: 985-995.

[40] Mao G, Zhang Z, Huang Z, Chen W, Huang G, Meng F, et al. MicroRNA-92a-3p regulates the expression of cartilage-specific genes by directly targeting histone deacetylase 2 in chondrogenesis and degradation. Osteoarthritis and Cartilage. 2017; 25: 521-532.

[41] Mao G, Zhang Z, Hu S, Zhang Z, Chang Z, Huang Z, et al. Exosomes derived from miR-92a-3p-overexpressing human mesenchymal stem cells enhance chondrogenesis and suppress cartilage degradation via targeting WNT5a. Stem Cell Research \& Therapy. 2018; 9: 247.

[42] Liu Q, Guo Y, Wang Y, Zou X, Yan Z. MiR-98-5p promotes osteoblast differentiation in MC3T3-E1 cells by targeting CKIP-1. Molecular Medicine Reports. 2018; 17: 4797-4802. 